\title{
Computerized Ultrasound Characterization of Breast Tumors
}

\author{
M. Alemán-Flores ${ }^{\mathrm{a}, *}$, P. Alemán-Flores ${ }^{\mathrm{b}}$, L. Álvarez-León ${ }^{\mathrm{a}}$, M.B.

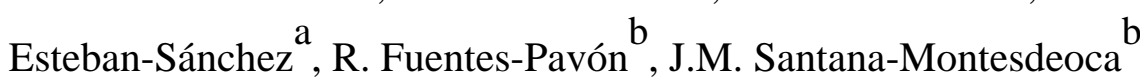 \\ ${ }^{a}$ Departamento de Informática y Sistemas, Universidad de Las Palmas de Gran Canaria, Spain \\ ${ }^{b}$ Sección de Ecografía, Servicio de Radiodiagnóstico, Hospital Universitario Insular de Gran Canaria, Spain
}

\begin{abstract}
In this paper, we present a new approach to the segmentation and analysis of solid breast nodules in ultrasonography. We have applied computer vision techniques to segment the nodules and analyze a series of diagnostic criteria which can help discriminate malignant and benignant tumors. The segmentation is carried out by means of a combination of a region growing algorithm and the active contour technique. On the other hand, the analysis of the diagnostic criteria involves several methods, such as the extraction of the minimum distance ellipse through gradient descent, pseudo-corner location or structure tensor. The methods which we propose have provided quite satisfactory results and show the usefulness of image processing techniques in the diagnosis by means of medical imaging. The aim of this work is not the substitution of the specialist, but the generation of a series of parameters which reduce the need of carrying out the biopsy.
\end{abstract}

Keywords: Breast cancer; Computer-aided diagnosis; Segmentation; Ultrasound; Active contours.

\section{Introduction}

The most reliable method to distinguish malignant from benign nodules is biopsy. Nevertheless, it is an expensive, time-consuming and uncomfortable technique, and its use must be reduced as much as possible. This is the main reason why specialists try to find a way to classify solid breast tumors using mammography or, as in our case, ultrasonography. Ultrasound images are very useful for the diagnosis and there are some factors observed in this kind of images which help the physicians to decide whether a certain tumor is malignant or benignant. However, speckle noise and the difficulty of the analysis of the diagnostic criteria make it very complex to extract an accurate and effective method for the segmentation of the nodules and the detection of such criteria.

\footnotetext{
* Corresponding author. E-mail address: maleman@dis.ulpgc.es.
} 
Currently, physicians analyze a series of factors directly on the ultrasound images and, according to the presence, absence and combination of different criteria, they determine whether the biopsy must be carried out or not. Among these criteria, hyperechogenicity, an ellipsoid shape, two or three gentle and well-circumscribed lobulations and a thin echogenic capsule are considered as benignity criteria. On the other hand, hypoechogenicity, a taller-than-wide shape, spiculation, angular margins, microlobulations, acoustic shadowing, microcalcifications and ramifications are considered as malignancy criteria [1].

The automatic analysis of ultrasonography can help the physicians in the discrimination by providing robust, objective and reproducible techniques and measures to classify the tumors according to their visual appearance in the ultrasound images. We have developed a series of techniques in order to obtain a common framework which allows extracting the contour of the tumor and carrying out a further analysis of the features that the specialists take into account when classifying the tumors. The main purpose consists in segmenting the nodules using a semi-automatic technique, extracting the regions of interest, according to the diagnostic criteria described above, and providing the specialists with some parameters which characterize the tumors and help decide whether they are malignant or benignant. Some other works deal with the location of the lesions [2], the analysis of general aspects [3][4], the comparison of different systems [5], or tissue analysis [6].

\section{Methods}

For the segmentation of the tumors, we have combined different computer vision techniques. First, an iterative implementation of the truncated median filter is applied to reduce speckle noise. Afterwards, the structure tensor is used to estimate the magnitude of the gradient in every point of the image. The specialist must select a point into the tumor and a threshold is set for the magnitude of the gradient, in such a way that a region-growing algorithm can be applied to extract an initial segmentation. The contour of this region is used as initial snake for a multiscale implementation of the active contour technique based on the following equation [7]:

$$
\frac{\partial u}{\partial t}=g_{\sigma}(I) \operatorname{div}\left(\frac{\nabla u}{\|\nabla u\|}\right)\|\nabla u\|+\lambda \nabla u \nabla g_{\sigma}(I)
$$

where $g_{\sigma}(I)$ is a stopping function given by:

$$
g_{\sigma}(I)=\frac{1}{\sqrt{1+\alpha\left\|\nabla I_{\sigma}\right\|^{2}}}
$$

The parameters of this active contour technique $(\alpha, \lambda$ and $\sigma$ ) are adapted at every scale to obtain more reliable results by adjusting the internal and external forces which guide 
the evolution of the snakes. Once the final contour is obtained, the specialist is allowed to correct the segmentation.

The further analysis of the tumor and the surrounding areas involves different types of techniques. For example, the general shape of the nodule is analyzed by fitting the contour to an ellipse. The distance from the contour to the ellipse is minimized using a gradient descent method in which five parameters (two coordinates for the center, two dimensions for the axes and an orientation angle) are adapted. The extraction of the minimum distance ellipse allows locating and measuring the ramifications of the nodule. In order to prevent the ramifications from modifying the central ellipse, an iterative process is applied in which the most distant points are eliminated and the ellipse is recalculated, so that the final ellipse represents the central part of the nodule. When the contour cannot be approximated to an ellipse, we search for two or three ellipses which can describe the contour in order to find gentle and well circumscribed lobulations. The global dimensions of the segmentation indicate the regions where the nodule is likely to be growing vertically.

On the other hand, the local examination of the contour allows analyzing certain features which involve the application of several computer vision techniques. For instance, the location of angular margins requires the extraction of pseudo-corners through line fitting. Those points with join two lines with an acute angle are considered as angular margins. The adjustment of contour segments to arcs allows finding the microlobulations. The measurement of the variation of gradient orientation indicates the degree of spiculation.

Finally, some other criteria are related to the contrast and mean intensity of certain areas, such as the segmented nodule, its capsule or the region under the nodule. These allow measuring the echogenicity, the calcifications, the intensity of the capsule and the acoustic shadowing.

Once the factors have been analyzed, some measures are provided, such as mean distance from the ellipse to the contour, the number of relevant elements (e.g. microlobulations, angular margins), contrast of calcifications, and some other parameters, in such a way that the benignity or malignancy of a tumor can be predicted in a more accurate way.

\section{Results}

The use of different filters and the combination of a region growing algorithm and a multiscale implementation of active contours have provided an effective way for the extraction of the contours. Fig. 1 shows an example of an initial ultrasound image of a breast nodule, the filtered image using the truncated median filter, the magnitude of gradient estimation through the structure tensor, the initial segmentation, the initial snake, and the final snake using the multiscale implementation of the active contour technique. The segmentation provided by the region-growing algorithm has been used as initial approximation for snake evolution. The comparison with the contours delineated by the specialists in a series of test nodules proves the reliability of the final segmentations. However, due to the difficulty of this kind of images, the radiologist is always allowed to modify the contour in order to correct the errors of the segmentation. Once it has been validated, the diagnostic criteria are analyzed. 

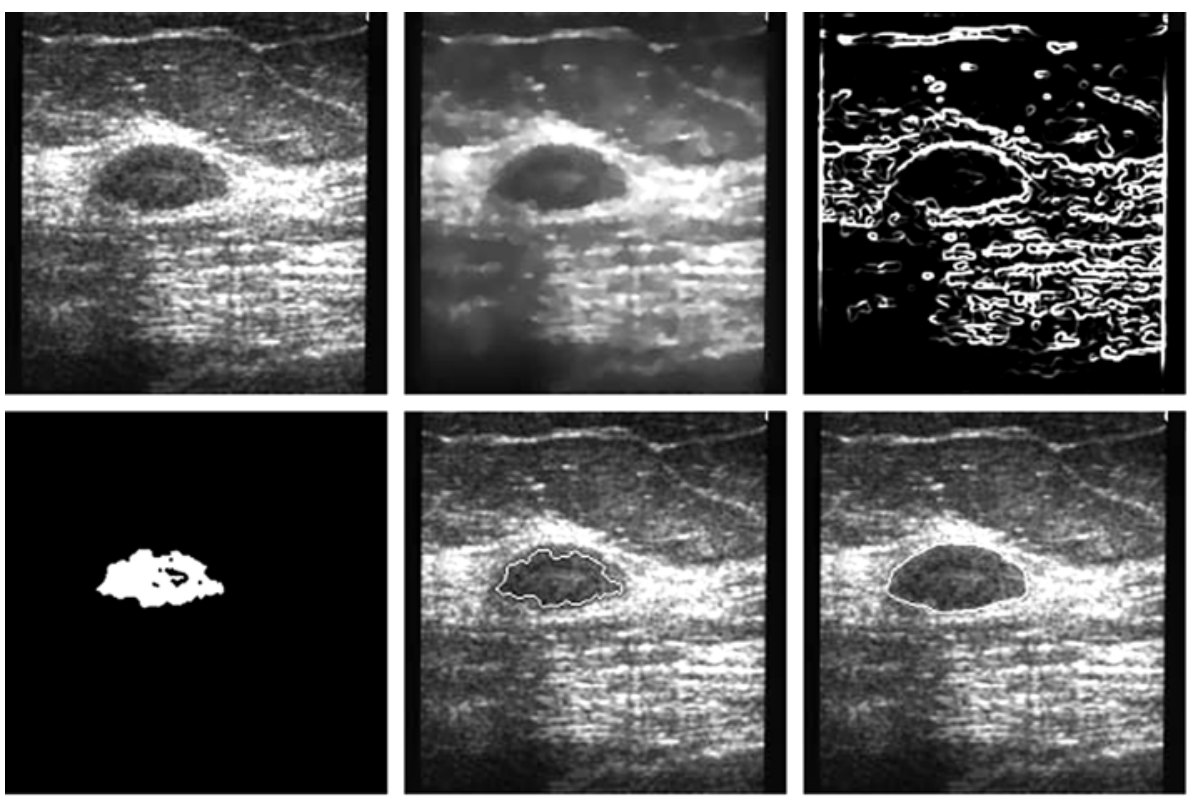

Fig. 1. From top to bottom and from left to right, an example of an ultrasound image of a nodule, the filtered image using the truncated median filter, the magnitude of gradient estimation through the structure tensor, the initial segmentation, the initial snake, and the final snake using the multiscale implementation of active contours.

The analysis of different features in the region of interest allows discriminating malignant and benignant tumors with a high precision. Fig. 2 shows the segmentation, the contour and the minimum distance ellipse for a breast nodule. Fig. 3 shows a nodule for which the minimum distance ellipse has been extracted. The iterative algorithm described above has been used to find the ellipse which best fits the central region of the nodule. This allows identifying and measuring the ramifications. In Fig. 4, even if the nodule is not quite elliptical, two gentle and well-circumscribed lobulations have been found, which is considered as a benignity criteria. Besides, the calcifications (small white and highly contrasted areas inside the nodule) have been marked. Fig. 5 shows the minimum distance ellipse and the microlobulations for a breast nodule.
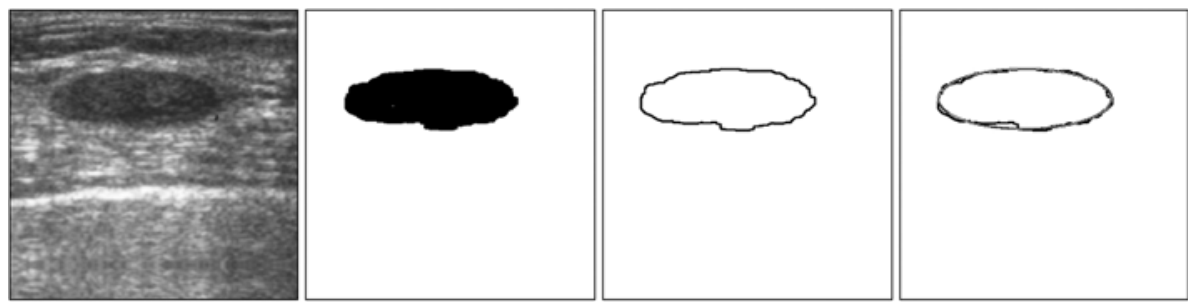

Fig.2. Original ultrasound image, segmentation, contour and minimum distance ellipse. 

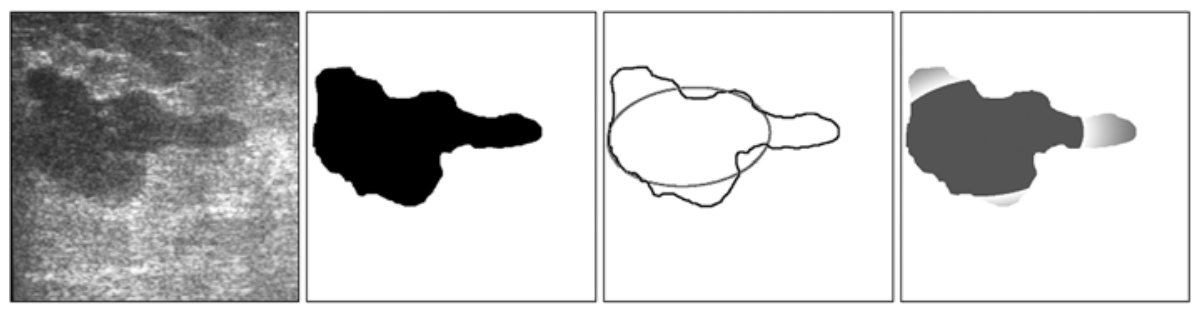

Fig.3. Original ultrasound image, segmentation, minimum distance ellipse and ramifications.
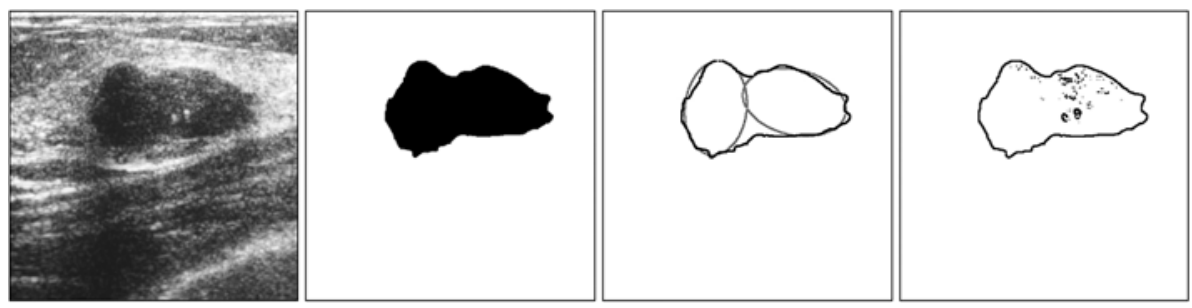

Fig.4. Original ultrasound image, segmentation, two gentle lobulations and calcifications.
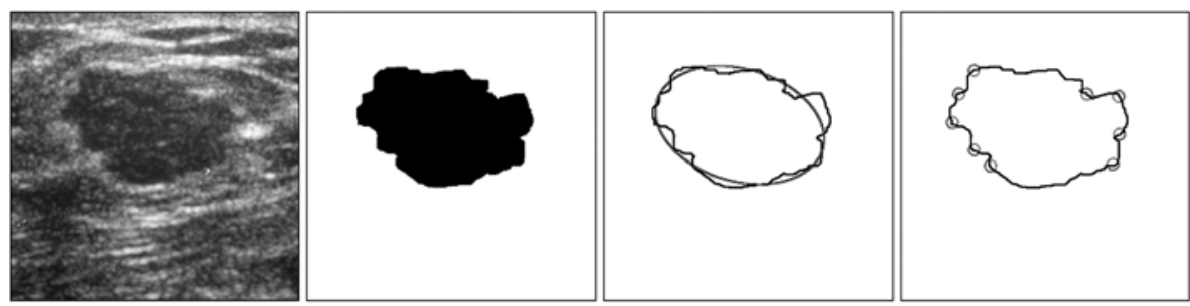

Fig.5. Original ultrasound image, segmentation, minimum distance ellipse and microlobulations.

The aim of this application is the location of those regions where the diagnostic criteria are likely to be present. This makes the decision making process much easier and objective.

\section{Conclusion}

We have introduced the basis for a new approach in the semi-automatic analysis of ultrasound images in order to provide a better understanding of breast ultrasound. The robustness and reproducibility of the methods, combined with the supervision of the specialists, try to increase the ratio of properly classified tumors without requiring the performance of a large number of biopsies.

The semi-automatic extraction of tumor contours by means of a combination of different filters and techniques has produced quite satisfactory segmentations of breast nodules. Moreover, the extraction of numerical measures and the location of the regions of interest in the diagnosis of the tumors increase the capability of distinguishing between benign and malignant lesions. The wide range of parameters which have been 
studied and the accuracy of the techniques which have been applied supply a complete analysis of solid breast nodules. The combination of the different criteria to provide more representative measurements and the adjustment of the parameters will increase the sensibility and specificity of the discrimination.

The results show the usefulness of computer vision techniques, not to substitute the appreciation of the physicians, but to introduce new parameters in order to improve the diagnosis and reduce the number of biopsies.

\section{References}

[1] A.T. Stavros, D. Thickman, C.L. Rapp, M.A. Dennis, S.H. Parker, G.A. Sisney, Solid breast nodules: use of sonography to distinguish between benign and malignant lesions, Radiology 196 (1995) 123-134.

[2] K. Drukker, M.L. Giger, K. Horsch, M.A. Kupinski, C.J. Vyborny, Computerized lesion detection on breast ultrasound. Medical Physics 29:7 (2002) 1438-1446.

[3] C.M. Cheng, Y.H. Chou, K.C. Han, et al., Breast Lesions on sonograms: Computer-aided diagnosis with nearly setting-independent features and artificial neural networks, Radiology 226 (2003) 504-514.

[4] D.R. Chen, R.F. Chang, Y.L. Juang, Computer-aided diagnosis applied to US of solid breast nodules by using neural networks, Radiology 213 (1999) 407-412.

[5] W.J. Kuo, R.F. Chang, W.K. Moon, C.C. Lee, D.R. Chen, Computer-aided diagnosis of breast tumors with different US systems, Acad Radiol 9 (2002) 793-799.

[6] J. Kaufhold, R. Chan, W.C. Karl, D.A. Castanon, Ultrasound tissue analysis and characterization, Battlefield Biomedical Technologies, H.H. Pien editor, Proc. SPIE V 3712 (1999).

[7] V. Caselles, R. Kimmel, G. Sapiro, Geodesic active contours, International Journal of Computer Vision 22:1 (1997) 61-79. 